\title{
Dye-sensitized solar cells using an anion-conducting polymer as a material of solid-state electrolyte
}

\author{
Yuki Ota, Takeo Hyodo, Yasuhiro Shimizu* \\ Graduate School of Engineering \\ Nagasaki University \\ 1-14 Bunkyo-machi, Nagasaki 852-8521, Japan \\ *shimizu@nagasaki-u.ac.jp
}

\author{
Hiroyuki Yanagi \\ Tokuyama Corporation \\ 40 Wadai, Tsukuba, Ibaraki 300-4247, Japan
}

\begin{abstract}
An anion-conducting polymer (ACP) has been used as an electrolyte material of solid-state dye-sensitized solar cells (DSCs) and the basic electrochemical properties of DSCs have been examined in this study. Initial photocurrent-voltage characteristics of the DSC prepared with ACP (DSC-S) were inferior to those of the DSC prepared with a general liquid electrolyte (DSC-Liq), but DSC-S exhibited better long-term stability. From AC impedance measurements, the relatively poor photocurrent-voltage characteristics of the as-fabricated DSC-S were anticipated to arise from larger resistance of charge transfer at electrolyte/ $\mathrm{TiO}_{2}$ electrode interface than that of the as-fabricated DSC-Liq.
\end{abstract}

Keywords; dye-sensitized solar cell; solid electrolyte; anionconducting polymer

\section{INTRODUCTION}

Dye-sensitized solar cells (DSCs) are a promising candidate as an alternative to conventional silicon-based solar cells, because of their low cost, relatively high energyconversion efficiency and a simple method for manufacturing, and the conversion efficiency of $11 \%$ for the DSCs has been achieved in some literatures [1, 2]. However, the long-term stability of the DSCs is extremely poor, because of leakage and volatilization of the liquid electrolyte involved. Hence, solidification of electrolyte is one of important techniques to improve these problems. Recently, several materials, such as

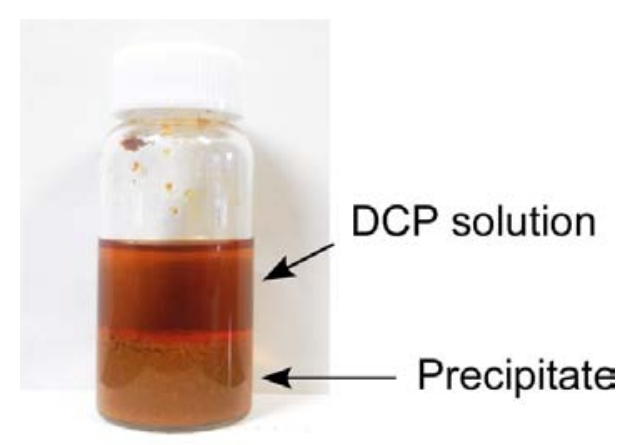

Figure 1. Photograph of precipitate deposited after the mixing of the ACP solution and the electrolyte solution.
CuSCN [3], 2,2',7,7'-tetrakis (N,N'-di(4-methoxyphenyl)amine)-9,9'-spirobifluorene (spiro-OMeTAD) [4], polyvinylidene difluoride (PVDF) [5], have been developed as a potential candidate of the solid-state electrolyte.

We have recently focused on anion-conducting polymers (ACP) as an electrolyte for various electrochemical devices, because of their high anion conductivity and improved longterm stability [6-8]. Therefore, ACP was employed as a solidstate electrolyte in fabricating DSCs, and fundamental electrochemical properties of the DSCs have been investigated in this study.

\section{EXPERIMENTAL}

\section{A. Preparation of $\mathrm{TiO}_{2}$ electrode modified with dye}

A commercial $\mathrm{TiO}_{2}$ powder (P-25, AEROXIDE ${ }^{\circledR}$ ) was mixed with appropriate amounts of surfactant (Triton-X 100, SIGMA-ALDRICH ${ }^{\circledR}$ ), pure water, polyethylene glycol (molecular weight: 20000) and acetylacetone. The slurry obtained was coated on an FTO substrate by spin coating (electrode area: $5 \times 5 \mathrm{~mm}^{2}$ ). After drying in air, it was heattreated at $500^{\circ} \mathrm{C}$ for $2 \mathrm{~h}$. Then, it was soaked in dye solution \{mixture of ruthenium dye $\left(\left[\mathrm{RuL}_{2}(\mathrm{NCS})_{2}\right]: 2 \mathrm{TBA}, \mathrm{L}: 2,2^{\prime}-\right.$ bipyridyl-4,4'-dicarboxylic acid, TBA: tetra-nbutylammonium (N-719), Solaronix), acetonitrile and tertbuthylalcohol $\}$ at $50^{\circ} \mathrm{C}$ for $12 \mathrm{~h}$ to ensure the adsorption of the dye on the surface of $\mathrm{TiO}_{2}$.

\section{B. Fabrication of solid-state DSC}

The mixture of $0.05 \mathrm{M} \mathrm{I}_{2}, 0.1 \mathrm{M} \mathrm{LiI,} 0.5 \mathrm{M}$ 4-tertbutylpyridine, $\quad 0.6 \mathrm{M}$ 1,2-dimethyl-3-propylimidazolium iodide and acetonitrile was used as electrolyte solution. The electrolyte solution and ACP solution (AS-4, Tokuyama Corp.), which is 1-propanol containing a 5 wt\% hydrocarbontype ionic polymer with quaternary ammonium salts (ionexchange capacity: $1.5 \mathrm{mmol} \mathrm{g}^{-1}$ and ion conductivity: ca. 13 $\mathrm{mS} \mathrm{cm}^{-1}$ ), were dropped on a dye-modified $\mathrm{TiO}_{2}$ film simultaneously, and they were dried at $50^{\circ} \mathrm{C}$. The volume ratio of the ACP solution and the electrolyte solution dropped was 3:2. In assembling a DSC, a spacer (masking tape) was set between a counter electrode (ITO glass substrate coated with platinum by magnetron sputtering) and the dye-modified $\mathrm{TiO}_{2}$ 
film which had been coated with the dried mixture of ACP and electrolyte components. The solid-state dye-sensitized solar cell thus fabricated was denoted as DSC-S.

However, a large amount of precipitate was deposited when the ACP solution was mixed with the electrolyte solution, as shown in Fig. 1. Thus, the supernatant liquid and the precipitate were separated and dried at $50^{\circ} \mathrm{C}$ for $1 \mathrm{~h}$. Viscosity of the supernatant liquid after drying was much larger than that before drying. The condensed supernatant liquid and the precipitate after the drying were coated on the dye-modified $\mathrm{TiO}_{2}$ electrode as an electrolyte of a DSC, respectively. The additional two kinds of DSCs were fabricated be using these $\mathrm{TiO}_{2}$ electrodes thus prepared, the separator and the counter electrode. The DSCs fabricated with

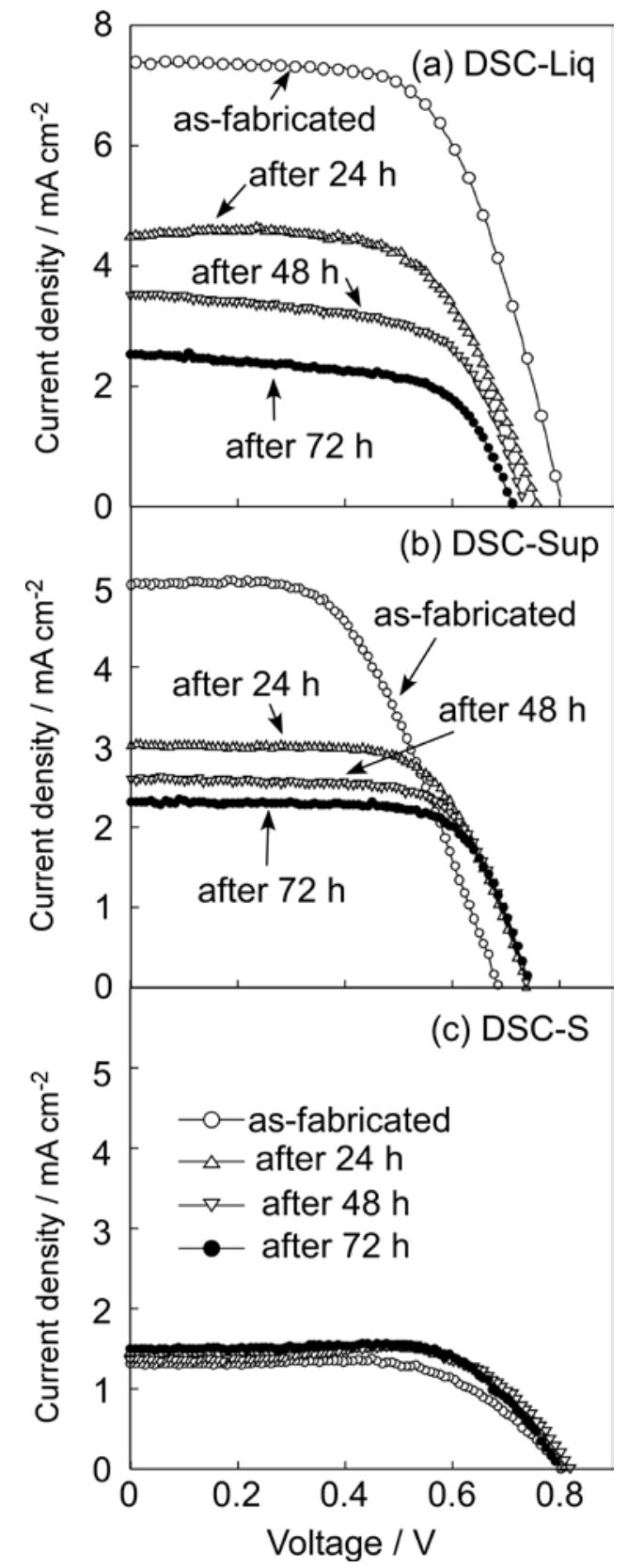

Figure 2. $J-V$ characteristics of (a) DSC-Liq, (b) DSC-Sup and (c) DSC-S. the condensed supernatant liquid and the precipitate were denoted as DSC-Sup and DSC-Pre, respectively. For comparative purpose, another DSC was also fabricated only with the electrolyte solution, and this is referred to as DSC-Liq.

\section{Characterization of DSCs}

The current density $(J)$-voltage $(V)$ measurements were performed under illumination of visible light generated from xenon lamp (100 $\mathrm{mW} \mathrm{cm}^{-2}$, cut with visible-light transmission filter). AC impedance characteristics of DSCs under open circuit conditions were also measured upon the illumination of visible light in the frequency range of $100 \mathrm{mHz} \sim 200 \mathrm{kHz}$ and at an AC amplitude of $10 \mathrm{mV}$.

\section{RESULTS AND DISCUSSION}

Figure 2 shows variations in $J-V$ characteristics of DSCLiq, DSC-Sup and DSC-S with time during their storage at RT under atmospheric conditions. Figure 3 shows variations in conversion efficiency $(\eta)$ of DSCs with storage time. $\eta$ is calculated by using the following equation:

$$
\eta=\left(\mathrm{P}_{\max } / \mathrm{I}\right) \times 100
$$

where $\mathrm{P}_{\max }$ is maximum power density, $\mathrm{I}$ is intensity of incident light. Since current density of as-fabricated DSC-Pre was extremely small over the whole voltage range, the $J-V$ characteristics of DSC-Pre could not be evaluated at any storage periods. As-fabricated DSC-Liq showed the largest short-circuit current density $\left(J_{\mathrm{sc}}\right.$, ca. $\left.7.40 \mathrm{~mA} \mathrm{~cm}{ }^{-2}\right)$ and $\eta$ (ca. $3.68 \%$ ) with relatively large open circuit voltage ( $V_{\text {oc }}$, ca. 0.80 $\mathrm{V})$, among all the DSCs tested. The $J_{\mathrm{sc}}$ of as-fabricated DSCSup (ca. $5.05 \mathrm{~mA} \mathrm{~cm}^{-2}$ ) was slightly smaller than that of the as-fabricated DSC-Liq, while the $V_{\text {ос }}$ value (ca. $0.68 \mathrm{~V}$ ) was the smallest among all the DSCs tested. On the other hand, asfabricated DSC-S showed the smallest $J_{\mathrm{sc}}$ (ca. $1.31 \mathrm{~mA} \mathrm{~cm}{ }^{-2}$ ) and $\eta$ (ca. $0.50 \%$ ), while the $V_{\text {ос }}$ value (ca. $0.80 \mathrm{~V}$ ) was comparable to that of DSC-Liq. The cell performances of DSC-Liq and DSC-Sup apparently reduced with an increase in the storage period at room temperature (RT) under

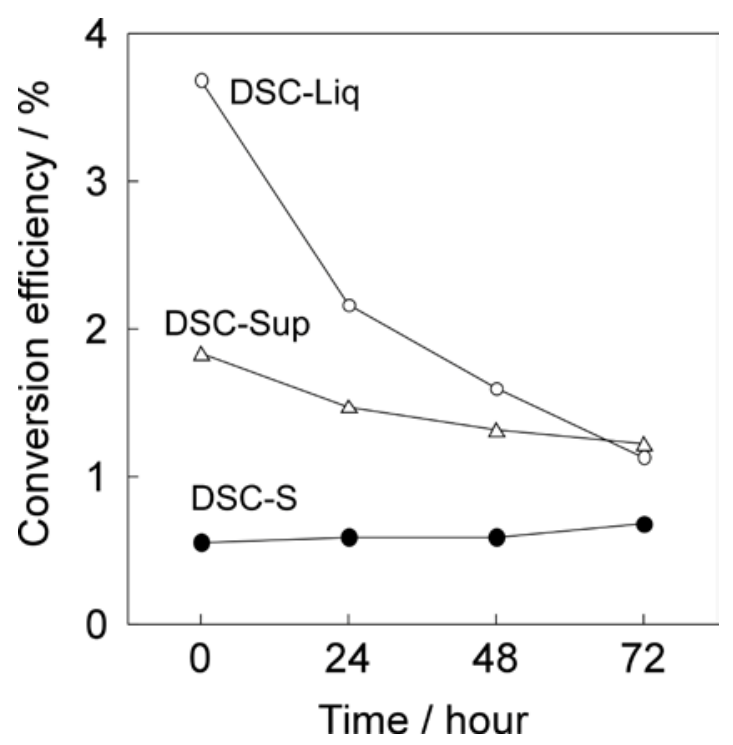

Figure 3. Variations in conversion efficiency of DSCs with storage time. 


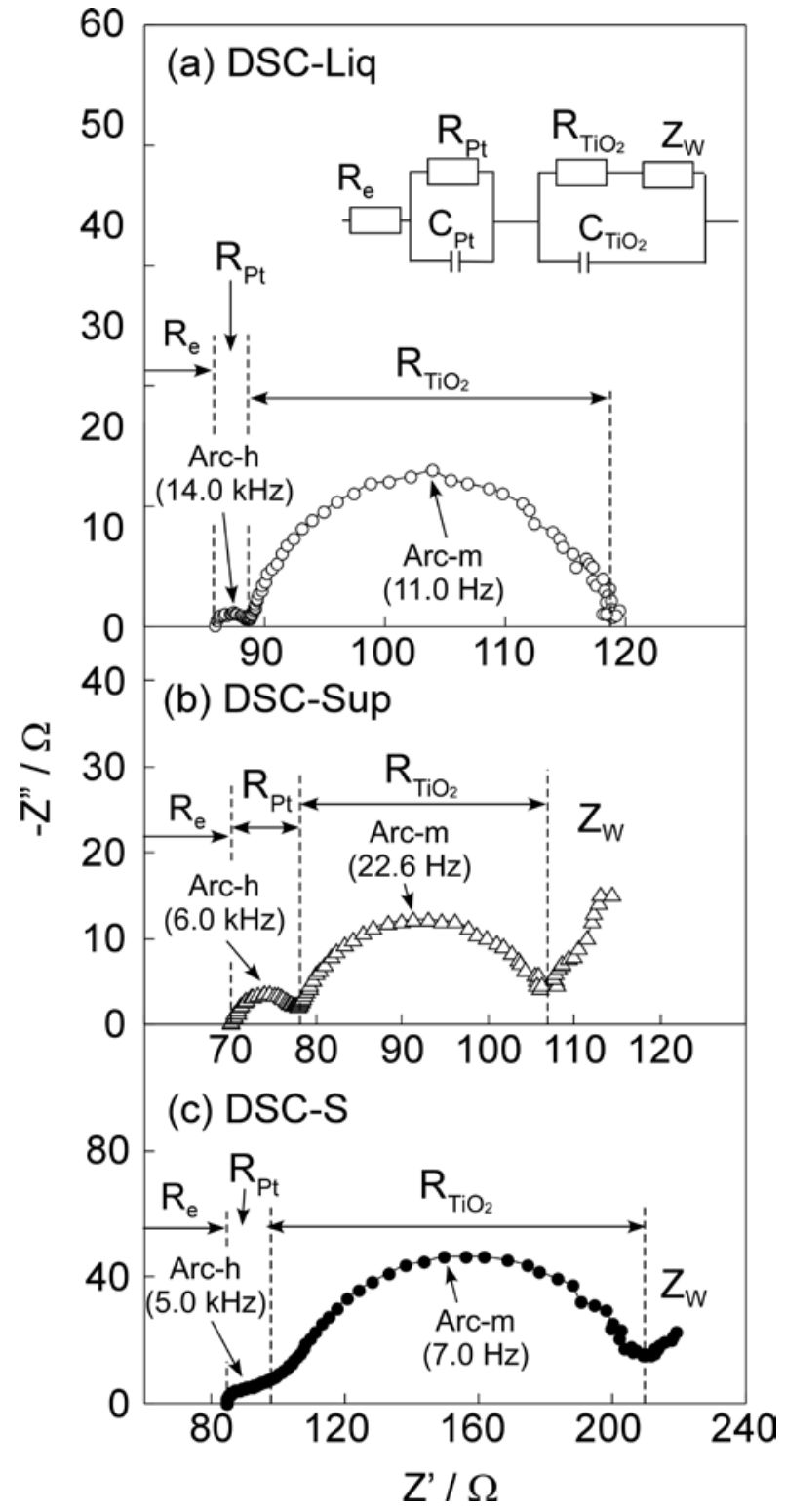

Figure 4. Nyquist plots of (a) DSC-Liq, (b) DSC-Sup and (c) DSC-S.

atmospheric conditions, and the $J_{\mathrm{sc}}$ values of DSC-Liq and DSC-Sol after $72 \mathrm{~h}$ was ca. 2.53 and $2.32 \mathrm{~mA} \mathrm{~cm}$, respectively.

On the other hand, the $J_{\text {sc }}$ and $\eta$ values of DSC-S remained unchanged, irrespective of prolonging the storage time. These results indicated that the DSC-S showed the most excellent long-term stability among the DSCs fabricated.

AC impedance characteristics of these as-fabricated DSCs were measured at RT, as shown in Fig. 4. As for all the DSCs, two kinds of arcs were confirmed in high $(\sim \mathrm{kHz})$ and medium $(\sim \mathrm{Hz})$ frequency ranges, and they are referred to as Arc-h and Arc-m, respectively. In addition, both DSC-Sup and DSC-S had another complex impedance component in a low frequency range $(\sim \mathrm{mHz})$.

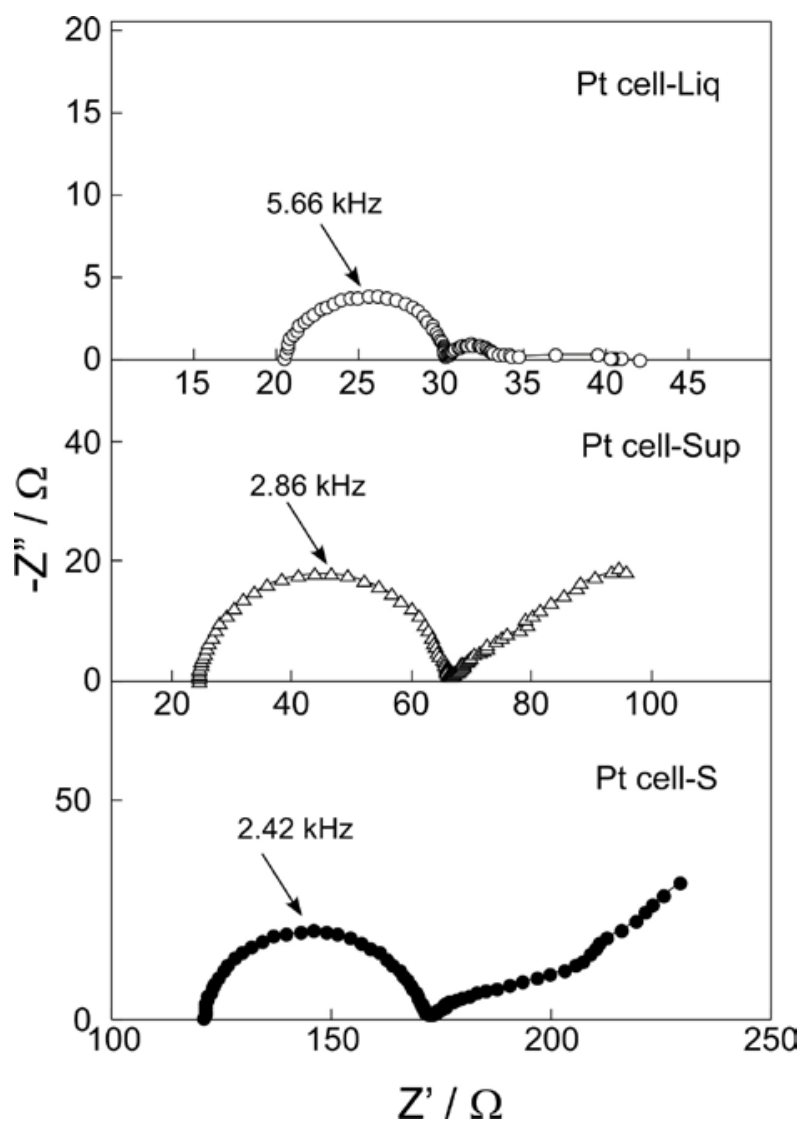

Figure 5. Nyquist plots of Pt cells.

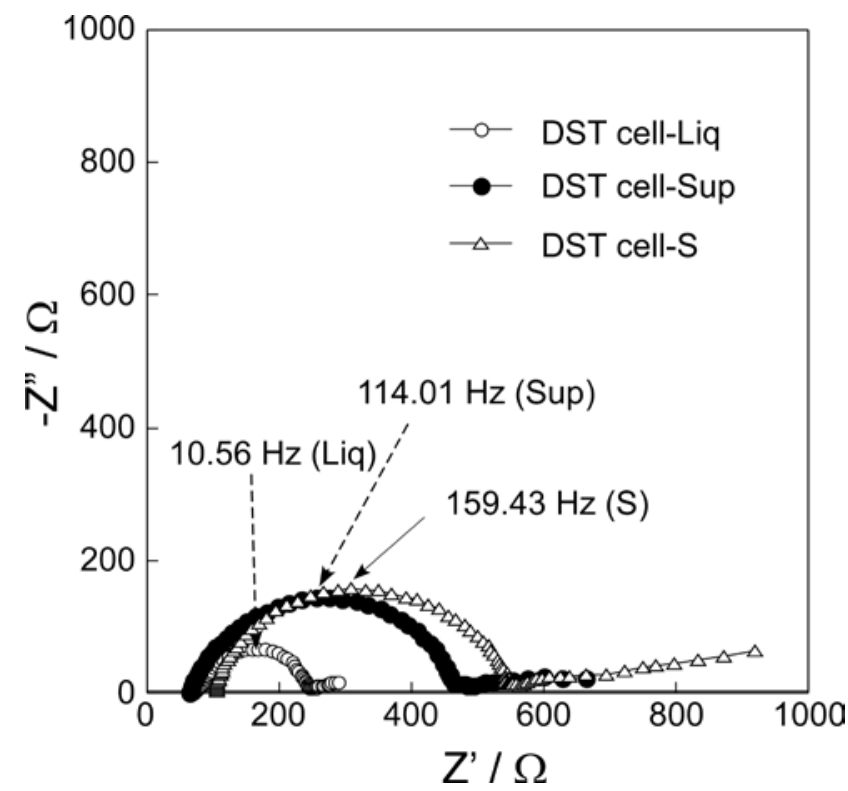

Figure 6. Nyquist plots of DST cells.

To get information on such components in the AC impedance characteristics of the DSCs, electrolytic cells with a pair of Pt electrodes or a pair of dye-modified $\mathrm{TiO}_{2}$ electrodes (Pt cell-N or DST cell-N, N: Liq, Sup or S) were fabricated and 
TABLE I. Results of impedance measurements.

\begin{tabular}{|c|c|c|c|c|}
\hline \multicolumn{2}{|c|}{ Component Cell } & DSC-L & $\begin{array}{l}\text { DSC- } \\
\text { Sup }\end{array}$ & DSC-S \\
\hline \multicolumn{2}{|r|}{$\mathrm{R}_{\mathrm{e}}(\Omega)$} & 85.8 & 70.2 & 84.5 \\
\hline \multirow{2}{*}{ Arc-h } & $\mathrm{R}: \mathrm{R}_{\mathrm{Pt}}(\Omega)$ & 2.3 & 7.6 & 15.6 \\
\hline & $C: C_{P t}\left(10^{-6} \mathrm{~F}\right)$ & 4.9 & 3.5 & 2.0 \\
\hline \multirow{2}{*}{ Arc-m } & $\mathrm{R}: \mathrm{R}_{\mathrm{TiO}_{2}}(\Omega)$ & 30.0 & 27.9 & 110.0 \\
\hline & $\mathrm{C}: \mathrm{C}_{\mathrm{TiO}_{2}}\left(10^{-4} \mathrm{~F}\right)$ & 4.8 & 2.5 & 2.1 \\
\hline \multicolumn{2}{|r|}{$Z_{w}(\Omega)$} & - & 50.5 & 113.9 \\
\hline
\end{tabular}

AC impedance characteristics of the Pt cell-N and the DST cell-N were measured at RT with illumination of visible light generated from xenon lamp (100 $\mathrm{mW} \mathrm{cm}^{-2}$, cut with visiblelight transmission filter), respectively, as shown in Figs. 5 and 6. As for all the Pt cells (Fig. 5), only one arc appeared in the high frequency range, and the size of the arc increased in the following order, Pt cell-Liq $<$ Pt cell-Sup $<$ Pt cell-S. The values of capacitance component calculated from these arcs by using the Eq. (2) were in the range of $\sim 10^{-6} \mathrm{~F}$ and they are comparable to those of capacitance component calculated Arc$h$ of the DSCs (see TABLE I ).

$$
2 \pi \mathrm{fRC}=1
$$

where $\mathrm{f}$ is a value of frequency at the top of the arc, $\mathrm{R}$ is a resistance value equal to the diameter of the arc. Therefore, the Arc-h of DSCs is considered to arise from the charge transfer resistance and capacitance of the $\mathrm{Pt}$ electrode/electrolyte interface. The size difference between the arc of Pt cells and the Arc-h of DSCs may be ascribed to slight variations in the experimental conditions. Another complex impedance component indicating Warburg impedance was confirmed for both the Pt cell-Sup and Pt cell-S in the low frequency range, while such a component was hardly seen for Pt cell-Liq. On the other hand, DST cells had only an arc in the medium frequency range. However, the size of the arcs was larger than that of the Arc-m of all the DSCs, and the values of frequency at the top of arcs of DST cell-Ns (N: Sup and S) were larger than those of the Arc-m of DSC-N (N: Sup and S), respectively, indicating the certain changes in both resistance and capacitance component values. Such differences may arise from a slight change in illumination conditions form one to another cell, leading to a change in redox behavior of $\mathrm{I}_{2} / \mathrm{I}_{3}^{-}$at the electrode/electrolyte interface. Actually, the values of resistance calculated frorm the arcs of DST cell-Sup and $-S$ are in the range of $300-400 \Omega$. Therefore, these values are closer to those calculated from the Arc-m of DSCs, rather than those of the Arc-h of DSCs (see TABLE I ). Thus, the Arc-m of DSCs can be considered to present the charge transfer resistance and capacitance of the dye-modified $\mathrm{TiO}_{2}$ electrode/electrolyte interface. Another complex impedance component, i.e. Warburg impedance, was also confirmed for all the DST cells in the low frequency range.

Based on these fundamental data, a possible equivalence circuit of DSCs is proposed as shown in the inset in Fig. 4.

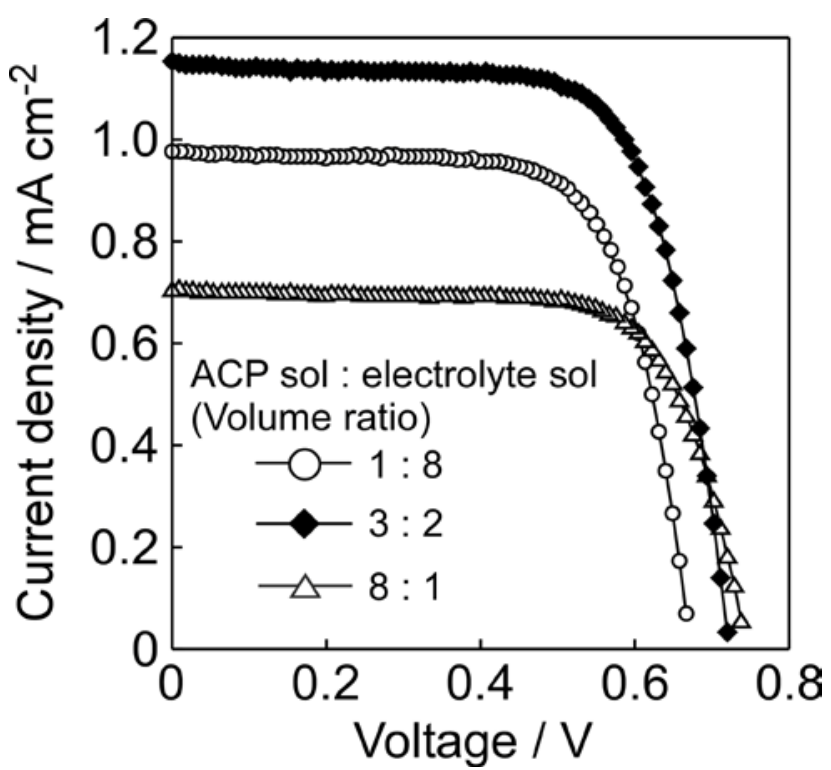

Figure 7. $J-V$ characteristics of DSC-Ss fabricated by using solid-state electrolytes prepared at different mixing ratios.

Here, $\mathrm{R}_{\mathrm{e}}, \mathrm{R}_{\mathrm{Pt}}$ and $\mathrm{R}_{\mathrm{TiO}_{2}}$ represent resistance of the electrolyte, charge transfer resistance on the Pt electrode, charge transfer resistance on the dye-modified $\mathrm{TiO}_{2}$ electrode, respectively, $\mathrm{Z}_{\mathrm{W}}$ stands for Warburg impedance including mass transfer effects in the diffusion layer near the electrode surface. $\mathrm{C}_{\mathrm{Pt}}$ and $\mathrm{C}_{\mathrm{TiO}_{2}}$ represent double layer capacitances on the Pt electrode and the dye-modified $\mathrm{TiO}_{2}$ electrode, respectively. In Nyquist plots shown in Fig. 4, therefore, Arc-h and Arc-m are determined to be ion relaxations on the Pt electrode and dyemodified $\mathrm{TiO}_{2}$, respectively. The electrolyte resistance can be obtained as a distance between the origin and the intercept point, which is the closest to the origin, of Arc-h. Values of the each component in the equivalence circuit are listed in TABLE I.

In Fig. $4, \mathrm{Z}_{\mathrm{W}}$ was observed in the low frequency range ( $\mathrm{mHz}$ ) only for DSC-Sup and DSC-S, and the $\mathrm{Z}_{\mathrm{W}}$ value of DSC$S$ was larger than that of DSC-Sup. Therefore, it was confirmed that the mass transfer rate in the diffusion layer near the surface of both the dye-modified $\mathrm{TiO}_{2}$ electrode and the $\mathrm{Pt}$ electrode of DSC-S was much slower than that of DSC-Liq. There was little difference in $\mathrm{R}_{\mathrm{e}}$ value among three DSCs in Fig. 4, irrespective of the appearance of large differences in Figs. 5 and 6 . Therefore, $\mathrm{R}_{\mathrm{e}}$ may contain additional contact resistance between DSCs and lead wires. On the other hand, $\mathrm{R}_{\mathrm{Pt}}$ and $\mathrm{R}_{\mathrm{TiO}_{2}}$ values of DSC-S were larger than that of other two DSCs. These results indicate that the charge transfer speed of ions at the electrodes was the slowest when the electrodes were contacted with the solid polymer.

Several DSC-Ss were also fabricated by using solid-state electrolytes prepared at different mixing ratios (ACP solution : electrolyte solution $=1: 8$ or $8: 1$ in volume), and effects of the mixing ratios on $J-V$ characteristics of as-fabricated DSCSs were measured. The $J-V$ curves of the additionally fabricated DSC-Ss were shown in Fig. 7, together with that of the original DSC-S (ACP solution: electrolyte solution $=3: 2$ 
in volume). The performances of the additionally fabricated DSC-Ss were poor than that of the original DSC-S.

These results reveal that the mixing ratio of the ACP and electrolyte-components also largely influences on the performance. Therefore, solid-state DSCs can be further improved by simultaneous optimization of the electrode/electrolyte interface conditions and the electrolyte composition.

\section{CONCLUSIONS}

A solid-state dye-sensitized solar cell (DSC-S) has been fabricated by using ACP as an electrolyte material, and its $J-V$ characteristics and AC impedance properties have been investigated in this study. The $J-V$ characteristics of asprepared DSC-S were relatively inferior to as-prepared DSCLiq and DSC-Sup, but the long-term stability was much better than that of DSC-Liq and DSC-Sup. In AC impedance measurements, the DSC-S showed the largest $R_{P t}$ and $R_{\mathrm{TiO}_{2}}$ values, indicating the slowest charge transfer rate of ions at the electrodes in the case of the direct contact with the solid polymer.

\section{REFERENCES}

[1] M. Grätzel, "Photoelectrochemical cells,” Nature, vol. 414, pp. 338-344, 2001.

[2] S. J. Lim, Y. Soo Kang, and D. W. Kim, "Rubbery copolymer electrolytes containing polymerized ionic liquid for dye-sensitized solar cells," Electrochim. Acta, vol. 56, pp. 2031-203, 2011.

[3] E. V. A Premalal, N. Dematage, G. R. R. A. Kumara, R.M.G. Rajapakse, M. Shimomura, K. Murakami, A. Konno, "Preparation of structurally modified, conductivity enhanced-p-CuSCN and its application in dyesensitized solid-state solar cells,” J. Power Sources, pp. 288-296, 2012.

[4] I.-K. Ding, N. Tetreault, J. Brillet, B. E. Hardin, E. H. Smith, S. J. Rosenthal, F. Sauvage, M. Grätzel, " Pore-Filling of Spiro-OMeTAD in Solid-state Dye Sensitized Solar Cells: Quantification, Mechanism, and Consequences for Device Performance,” M. D. McGehee, Adv. Funct. Mater, vol. 203 19, 2431-2436, 2009.

[5] K. C. Huang, P. Y. Chen, R. Vittal, K. C. Ho, "Enhanced performance of a quasi-solid-state dye sensitized solar cell with aluminum nitride in its gel polymer electrolyte,” Solar Energy Mater. \& Solar Cells,

[6] H. Yanagi and K. Fukuda, "Anion Exchange Membrane and Ionomer for Alkaline Membrane Fuel Cells,” ECS Transactions, 16 (2008) 257.

[7] T. Hyodo, C. Ishibashi, K. Matsuo, K. Kaneyasu, H. Yanagi and Y. Shimizu, "CO and $\mathrm{CO}_{2}$ Sensing Properties of Electrochemical Gas Sensors Using an Anion-conducting Polymer as an Electrolyte," Electrochim. Acta, in press.

[8] H. Yanagi and K. Fukuda, "Anion Exchange Membrane and Ionomer for Alkaline Membrane Fuel Cells,” ECS Transactions, 16 (2008) 257. 